\title{
Halogen Effect on the Photodissociation Mechanism for Gas-Phase Bromobenzene and lodobenzene
}

\author{
Xiao-Peng Zhang, ${ }^{[a, b]}$ Zheng-Rong Wei, ${ }^{[c]}$ Ying Tang, ${ }^{[c,+]}$ Ting-Jung Chao, ${ }^{[a, b]}$ Bing Zhang, ${ }^{[c]}$ \\ and King-Chuen $\operatorname{Lin}^{*[a, b]}$
}

\begin{abstract}
The velocity imaging technique combined with $(2+1)$ resonanceenhanced multiphoton ionization (REMPI) is used to detect the halogen fragments in the photodissociation of bromobenzene and iodobenzene at $266 \mathrm{~nm}$. With the aid of potential energy curve calculations by Lunell (Y. J. Liu, P. Persson, S. Lunell, J. Phys. Chem. A 2004, 108, 2339-2345.), the Br fragmentation is proposed to stem from excitation of the lowest excited singlet $\left(\pi-\pi^{*}\right)$ state followed by predissociation along a repulsive triplet $\left(n-\sigma^{*}\right)$ state. The slowed dissociation rate leads to production of the isotropic $\mathrm{Br}$ fragments and $93 \%$ internal energy deposition. Only the ground state $\operatorname{Br}\left({ }^{2} P_{3 / 2}\right)$ is detectable. In contrast,
\end{abstract}

when iodine is substituted, the iodine effect stabilizes the repul sive states associated with the $\mathrm{I}-\mathrm{C}_{6} \mathrm{H}_{5}$ bond rupture and the subsequent dissociation channels become more complicated. $84 \%$ of the iodobenzene molecules obtained follow a direct dissociation channel, while the remaining undergo a predissociative process. Both routes result in rapid dissociation with anisotropy parameters of $0.7 \pm 0.2$ and $0.9 \pm 0.2$ as well as $70 \%$ and $26 \%$ in the fractions of translational energy deposition, respectively. The relative quantum yields of $I^{*}$ and I are 0.35 and 0.65 and their related photodissociation pathways are discussed in detail.

\section{Introduction}

During photodissociation of halogenated molecules such as alkyl halides, irradiation with UV light causes rapid bond fission to release halogen atoms. The non-bonding electron of the halogen atom undergoes a transition to the antibonding $\sigma^{*}$ orbital localized on the $\mathrm{C}-\mathrm{X}$ bond (X denotes halogen). ${ }^{[1-8]}$ In contrast, the photodissociation processes for aryl halides are more complicated since more electronic states are involved, thus making multiple dissociation channels probable. ${ }^{[9-20]}$ These include predissociation via intersystem crossing (ISC), hot molecular dissociation via internal conversion, and direct photodissociation from a repulsive surface. The dissociation rates and channels depend substantially on the type of halogen, the substituents on the phenyl ring and their relative positions, as well as the excitation wavelengths. ${ }^{[9-20]}$

Among the aryl halides studied, the active focus has been on the simplest type of monohalobenzenes. In early work by Bersohn, ${ }^{[9,10]}$ these compounds were expected to follow a predissociation mechanism, initially excited to a bound singlet $\left(\pi-\pi^{*}\right)$ state which interacted with a repulsive triplet $\left(n-\sigma^{*}\right)$ state by spin-orbit coupling. In addition to the predissociation channel, a fast channel was found for chlorobenzene at 193 and $248 \mathrm{~nm},{ }^{[21]}$ and iodobenzene at $305 \mathrm{~nm} \cdot{ }^{[13]}$ The second channel was ascribed to direct photodissociation via the repulsive $\left(n-\sigma^{*}\right)$ state localized on the $C-X$ bond. Han and coworkers ${ }^{[16]}$ found only a fast photodissociation channel in the $266 \mathrm{~nm}$ photolysis of bromobenzene with a transition dipole moment lying perpendicular to the $\mathrm{C}-\mathrm{Br}$ bond. Kadi et al. ${ }^{[22]}$ reported two fast dissociation channels occurring in iodobenzene at $266 \mathrm{~nm}$, but only one in bromo- and chlorobenzene. They also found that the dissociation rates increase with increasing mass of the halogen atom, confirming involvement of ISC in the predissociation. The additional dissociation route observed in iodobenzene results from direct dissociation by excitation to an antibonding orbital $\left(n-\sigma^{*}\right)$, as reported previously. ${ }^{[9-11,13,14,23,24]}$ The threshold wavelength for the $\mathrm{F}-\mathrm{C}$ bond rupture in fluorobenzene is about $196 \mathrm{~nm} \cdot{ }^{[25]}$ Huang ${ }^{[26]}$ and Lee ${ }^{[27]}$ observed a substantial yield of HF via four-center elimination in the ground state and a minor hydrogen atom, instead of a fluorine atom, in photodissociations at 157 and $193 \mathrm{~nm}$. Substituent effects on the photodissociation of more complicated aryl halides has also been investigat$\mathrm{ed}_{,}^{[12,17,18,20,28,29]}$ including dibromobenzenes, tribromobenzene, $^{[19]}$ bromofluorobenzenes, ${ }^{[17,20]}$ chlorotoluenes, ${ }^{[12]}$ and bromotoluenes. ${ }^{[29]}$

Thus far, the femtosecond pump-probe technique ${ }^{[11,18-20,22]}$ and photofragment translational spectroscopy (PTS $^{[9,10,12-14,16,17,21,24,28,29]}$ have been the methods of choice in

\footnotetext{
[a] X.-P. Zhang, T.-J. Chao, K.-C. Lin Institute of Atomic and Molecular Sciences Academia Sinica, Taipei 106 (Taiwan) Fax: $(+886)$ 2-23621483

E-mail:kclin@ccms.ntu.edu.tw

[b] X.-P. Zhang, T.-J. Chao, K.-C. Lin Department of Chemistry National Taiwan University, Taipei 106 (Taiwan)

[c] Z.-R. Wei, Y. Tang, ${ }^{+}$B. Zhang State Key Laboratory of Magnetic Resonance and Atomic and Molecular Physics Wuhan Institute of Physics and Mathematics Chinese Academy of Sciences Wuhan 430071 (P. R. China)

${ }^{\left.{ }^{+}\right]}$Current address: Chemical Dynamics Lab., RIKEN Wako 351-0198 (Japan)
} 
photodissociation studies of aryl halides. Femtosecond laser spectroscopy provides information on dissociation rate constants for the related routes by detecting the parent molecular cations or fragment cations after the loss of a halogen atom. On the other hand, PTS gives insight into the fractions of translational and internal energies partitioned from the available energy and anisotropy parameters in the photolysis, by monitoring time-of-flight (TOF) mass spectra of the fragments at different laboratory angles.

As an alternative, velocity imaging detection coupled with resonance-enhanced multiphoton ionization (REMPI) has been used much less, except for iodobenzene. ${ }^{[15,30,31]}$ By using this technique, Gordon and coworkers ${ }^{[15]}$ focused on the influence of the above-threshold effect on the dissociative autoionization of the super-excited state of iodobenzene by multiphoton absorption. Poulsen et al. ${ }^{[30]}$ reported a radiation polarization effect on the iodine product yield of selective channels following photodissociation of iodobenzene at $266 \mathrm{~nm}$. Peronne et al. investigated alignment dynamics by measuring time-dependent angular distributions of the spatial orientation of the molecules. ${ }^{[31]}$

By means of the velocity imaging technique, knowledge of translational energy and angular distributions of the photofragments is extracted from the fragment images. The branching ratio of the spin-orbit ground and excited state halogen is also determined by selecting a particular wavelength in REMPI. By taking advantage of its merits, we investigate the photodissociation of bromobenzene and iodobenzene at $266 \mathrm{~nm}$ to probe the halogen effect. We observe only one dissociation channel for bromobenzene with an anisotropy parameter $\beta$ close to zero, suggesting that $\mathrm{Br}$ is isotropically fragmented. This stands in contrast to results obtained earlier with PTS. ${ }^{[16]}$ The photodissociation route is proposed to stem from predissociation by ISC. The ground state $\operatorname{Br}\left({ }^{2} \mathrm{P}_{3 / 2}\right)$ is the only bromine product detectable. For the photodissociation of iodobenzene, two dissociation channels with positive $\beta$ values and large fractions of translational energy deposition are observed, suggesting very short dissociation lifetimes in accord with those obtained by femtosecond laser spectroscopy. ${ }^{[11,22]}$ Both the I and $I^{*}$ fragments are found. With aid of ab initio calculations of one-dimensional potential energy curves, ${ }^{[25]}$ detailed photodissociation routes are discussed.

\section{Experimental Section}

The velocity imaging apparatus, similar to that used previously, ${ }^{\left[{ }^{[8]}\right.}$ consisted of a molecular beam source chamber and a main chamber, both of which were pumped to remain at a low pressure of $\sim 2 \times 10^{-7}$ Torr. Liquid samples of bromobenzene and iodobenzene (99\% purity) were used without further purification. Each was carried by helium gas at $1 \mathrm{~atm}$ through a pulsed valve with a $0.6 \mathrm{~mm}$ diameter orifice (General Valve Co.) operating synchronously with the laser pulses at $10 \mathrm{~Hz}$, and expanded into the source chamber. After passing through a $1 \mathrm{~mm}$ diameter skimmer and a collimator, the molecular beam was intersected perpendicularly by a linearly polarized laser beam in a two-stage ion lens region. The skimmer was mounted $30 \mathrm{~mm}$ downstream from the nozzle to divide the source chamber from the main chamber, which comprised an elec- trostatic lens, a time-of-flight (TOF) drift tube, and a two-dimensional position-sensitive detection system. Following the pioneering work of the ion velocity map imaging technique by Eppink and Parker, ${ }^{[32]}$ the set of electrostatic ion lenses was designed to consist of a repeller, an extractor, and ground electrodes, each with a central hole of 2,16 , and $16 \mathrm{~mm}$ in diameter, respectively. The biased voltages were optimized by simulation using a standard ion optics trajectory program (Simion 6.0). ${ }^{[33]}$

A $308 \mathrm{~nm} \mathrm{XeCl} \mathrm{excimer} \mathrm{laser-pumped} \mathrm{dye} \mathrm{laser} \mathrm{(FL3002,} \mathrm{Lambda}$ Physik) operating at $10 \mathrm{~Hz}$ was the only radiation source employed. Its output beam was frequency-doubled to emit at about $266 \mathrm{~nm}$ with an energy range of $40-200 \mu \mathrm{J}$ (AutoTrack III, InRad), followed by linear polarization perpendicular to the flight tube direction, and focused at the skimmed beam with a $200 \mathrm{~mm}$ focal-length lens. The bromobenzene and iodobenzene molecules were then photolyzed and the halogen-atom fragments were successively ionized by using the $(2+1)$ REMPI technique.

The resulting halogen ions were extracted and accelerated into a $36 \mathrm{~cm}$ long field-free drift tube along the molecular beam direction. The ion-cloud expansion was driven by both the fragment recoil velocity and a non-homogeneous electric field formed within the ion lens region, followed by projection onto a two-stage microchannel plate (MCP) and a phosphorus screen (FM3040, Galileo). The MCP could be gated within a minimum duration of $250 \mathrm{~ns}$ for mass selection. The ion imaging on the phosphorus screen was recorded by a charge-coupled device (CCD) camera (200XL4078, Pixelfly). The laser wavelength was scanned back and forth within the range of Doppler broadening to cover all the velocity components of the selected fragments. All the ion signals without gate restriction are acquired by a photomultiplier tube, instead of the CCD camera, and then transferred to a transient digitizer for display of the TOF mass spectrum.

\section{Results and Discussion}

\subsection{Photodissociation of Bromobenzene}

Upon irradiation of the laser source at near $266 \mathrm{~nm}$, bromobenzene dissociates to release the bromine fragments $\operatorname{Br}\left({ }^{2} \mathrm{P}_{3 / 2}\right)$ and $\operatorname{Br}^{*}\left({ }^{2} \mathrm{P}_{1 / 2}\right)$, which are successively ionized by $(2+1)$ REMPI at 266.55 and $266.61 \mathrm{~nm}$, respectively, and then captured with the velocity imaging detection system. For the first time the resultant raw ion image of $\mathrm{Br}$ is shown (Figure $1 \mathrm{a}$ ). This image is obtained by accumulating over 40000 laser shots with the background removed by subtracting a reference image collected at an off-resonance wavelength under the same conditions. a)

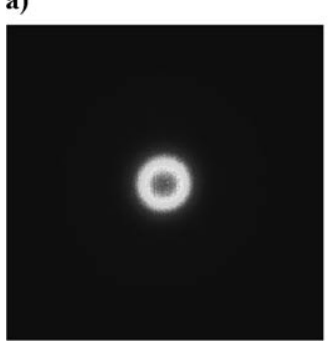

b)

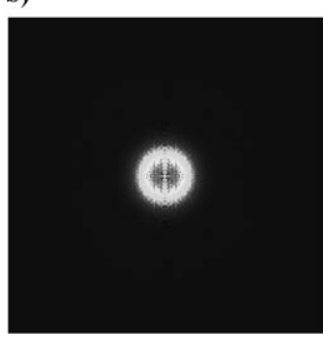

Figure 1. a) Raw ion image and b) inverse Abel-transformed ion image of the ground state $\mathrm{Br}$ for the photodissociation of bromobenzene at $266 \mathrm{~nm}$. The laser is linearly polarized in the vertical direction. 
The $\mathrm{Br}^{*}$ signals are not detectable, even though their images are accumulated over 100000 laser shots. The obtained raw image is a two-dimensional projection of the three-dimensional speed and angular distributions with cylindrical symmetry around the polarization axis of the photolyzing laser. According to the method of inverse Abel transformation, the corresponding three-dimensional spatial distributions of the fragments are reconstructed, as displayed in Figure $1 \mathrm{~b}$, exhibiting only one ring component.

The speed distribution $P(v)$ can be extracted by integrating the reconstructed three-dimensional speed distribution over all angles at each speed. The center-of-mass translational energy distribution $P\left(E_{T}\right)$ is obtained by converting the speed distribution using Equations (1) and (2)

$P\left(E_{\mathrm{T}}\right)=P(v) \frac{d v}{d E_{\mathrm{T}}}$

$E_{\mathrm{T}}=\frac{1}{2}\left(m_{\mathrm{Br}}+m_{\mathrm{C}_{6} \mathrm{H}_{5}}\right) \times \frac{m_{\mathrm{Br}}}{m_{\mathrm{C}_{6} \mathrm{H}_{5}}} \times v_{\mathrm{Br}}^{2}$

where $E_{\mathrm{T}}$ is the total translational energy; $m_{\mathrm{Br}}$ and $m_{\mathrm{C}_{6} \mathrm{H}_{5}}$ the respective masses of $\mathrm{Br}$ and $\mathrm{C}_{6} \mathrm{H}_{5}$, and $v_{\mathrm{Br}}$ the velocity of the bromine fragment. The available energy $E_{\text {avl }}$ for the dissociation process is evaluated by Equation (3)

$E_{\mathrm{avl}}=E_{\mathrm{hv}}-D_{0}-E_{\mathrm{el}}+E_{\mathrm{int}}$

where $E_{\mathrm{hv}}$ is the photon energy; $E_{\mathrm{el}}$ equal to $0 \mathrm{kcal} \mathrm{mol}^{-1}$ for the electronic energy of the ground state bromine atom, and $E_{\text {int }}$ the internal energy of the parent molecule, which is expected to be zero, since the rotational and vibrational excitations are negligible in a supersonic molecular beam. $D_{0}$ denotes the dissociation energy of the $\mathrm{C}-\mathrm{Br}$ bond, which is $81.6 \mathrm{kcalmol}^{-1}$ for bromobenzene, as determined by PTS. ${ }^{[17]}$ The fraction $f_{\mathrm{T}}$ of the translational energy deposition, defined as the ratio of the average translational energy to the available energy, is determined by Equation (4)

$f_{\mathrm{T}}=\frac{<E_{\mathrm{T}}>}{E_{\mathrm{avl}}}$

Figure 2 shows the center-of-mass translational energy distribution of the $\mathrm{Br}$ fragmentation from bromobenzene. Its profile can be fitted by one Gaussian curve. The position marked by the arrow indicates the maximum amount of kinetic energy available for one-photon dissociation of the parent molecule. The detected $\mathrm{Br}$ fragment apparently comes from molecular photodissociation, followed by ionization by $(2+1)$ REMPI. Since the dissociation lifetime of the excited state prepared at $266 \mathrm{~nm}$ is $26 \mathrm{ps}^{\left[{ }^{[22]}\right.}$ many of the excited molecules dissociate prior to absorbing an additional ns-pulsed photon for ionization. The process of dissociative ionization, that is, $\mathrm{C}_{6} \mathrm{H}_{5} \mathrm{Br}^{+} \rightarrow \mathrm{C}_{6} \mathrm{H}_{5}{ }^{+}+$

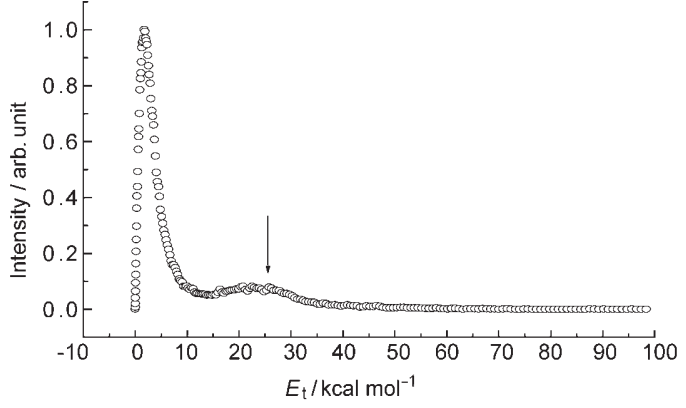

Figure 2. Center-of-mass translational energy distributions of the $\mathrm{Br}$ fragment produced from the photodissociation of bromobenzene at $266 \mathrm{~nm}$. The arrow indicates the maximum kinetic energy available for single-photon dissociation. Each data point $(\circ)$ corresponds to the relative signal intensity in arbitrary units.

$\mathrm{Br}$, should consume at least one more photon than the molecular dissociation. However, this is not supported by our findings. There is a tiny bump after the arrow mark (Figure 2), which is probably caused by the multiphoton effect. While considering the slow dissociation channel by one-photon absorption, the fraction $f_{\mathrm{T}}$ of the translational energy component is thus $7 \%$.

The fragment angular distribution $I(\theta)$ is obtained by integrating the reconstructed three-dimensional spatial distribution over a proper range of speed at each angle. It is characterized by an anisotropy parameter $\beta$, as expressed by Equation $(5)^{[34]}$

$I(\theta)=(4 \pi)^{-1}\left[1+\beta P_{2}(\cos \theta)\right]$

where $\beta$ is limited between 2 and $-1, \mathrm{P}_{2}(\cos \theta)$ is the secondorder Legendre polynomial, and $\theta$ is the angle between the laser polarization direction and the recoil velocity of fragments. $\beta$ is obtained by a least-squares fit to the angular distribution, and is $-0.02 \pm 0.01$ for photodissociation at $266 \mathrm{~nm} . \beta(\mathrm{Br})$ is approximately zero, indicating that the related dissociation channel is slow enough to produce the isotropic fragments. The parameters obtained are summarized in Table 1.

By using femtosecond laser spectroscopy with a $270 \mathrm{~nm}$ pump pulse, Davidsson and coworkers ${ }^{[20]}$ obtained a dissociation lifetime of $26 \mathrm{ps}$ for bromobenzene, which is longer than a molecular rotation period. With the aid of ab initio one-dimensional potential energy curves along the $\mathrm{C}-\mathrm{X}$ bond dissociation, ${ }^{[19,25]}$ they proposed that the slow dissociation is caused 
by predissociation via ISC from the lowest singlet excited state $\mathrm{S}_{1}\left[\left(\pi-\pi^{*}\right), \mathrm{B}_{2}\right]$ crossing to a repulsive triplet state, which correlates with the ground state bromine fragment. For clear interpretation, a simplified energy diagram for the molecular dissociation is shown in Figure 3. The energy at the $\mathrm{S}_{1}-\mathrm{T}_{4}$ crossing
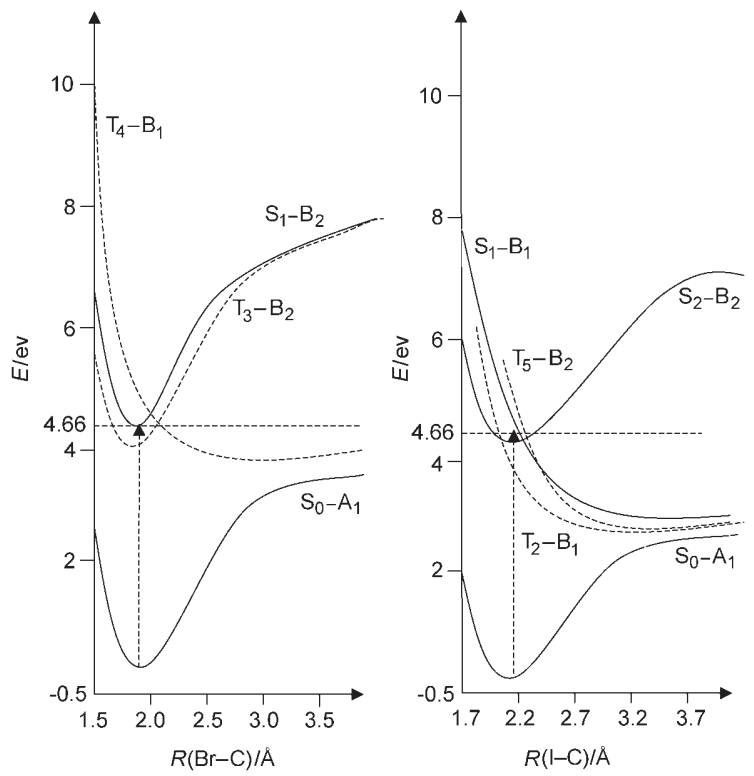

Figure 3. Simplified energy diagrams of one-dimensional potential energy curves along the $\mathrm{C}-\mathrm{X}(\mathrm{X}=\mathrm{Br}$ or $\mathrm{I})$ bond dissociation for a) bromobenzene and $b$ ) iodobenzene. Singlet states are represented by (-), while (-----) denotes triplet states. The excitation energy at $4.66 \mathrm{eV}$ by a laser source is denoted by a dashed vertical arrow. The detailed diagrams and calculations are adapted from ref. [25] by Lunell and co-workers.

point is $0.21-0.52 \mathrm{eV}$ higher than the $\mathrm{S}_{1}$ minimum state, depending on the method of calculation. ${ }^{[19]}$ Although one-dimensional potential energy curves are effective in predicting photodissociation pathways, multi-dimensional surface couplings are expected to be involved in the processes and this might change the activation barrier somewhat. For instance, in the photodissociation of bromo-3,5-difluorobenzene, the singlettriplet crossing barrier is quite reduced when reaction coordinate combines a $\mathrm{C}-\mathrm{Br}$ bond stretch and a $\mathrm{Br}$ out-of-plane bending on the $S_{1}$ surface. ${ }^{[35]}$ The spin-orbit transition is also facilitated when the upper state is a mixture of $\pi-\sigma^{*}$ and $n-\sigma^{*}$, since the coupling strength with only one-electron involvement is larger. ${ }^{[19,20,25,35]}$ Borg et al. ${ }^{[20]}$ recently assigned the first repulsive triplet state in bromofluorobenzenes to $\pi-\sigma^{*}$, which differs from most previous assignments in aryl halides. The slowing down of the predissociation rate by surpassing a barrier can lead to the production of the isotropic $\mathrm{Br}$ fragments. Thus, the $\beta(\mathrm{Br})$ value obtained herein lends support to this proposed mechanism. In addition, the slow predissociation process allows for more available energy being partitioned into the internal states of the fragment. Internal energy deposition of $93 \%$ is obtained.

In an earlier photodissociation study of the same compound, $\mathrm{Han}^{[16]}$ utilized photofragment translational spectroscopy to obtain $f_{\mathrm{T}}=47 \%$ and $\beta=-0.7 \pm 0.2$ in a $266 \mathrm{~nm}$ photolysis. They proposed two possible dissociation mechanisms: one is via direct dissociation along a repulsive surface $\left(n-\sigma^{*}\right)$ and the other is via fast predissociation which occurs if the parent molecules are excited to a bound $S_{1}$ state, crossing through a point that lies below the laser excitation energy to a repulsive triplet state. However, in ab initio calculations of one-dimensional potential energy curves, ${ }^{[19,25]}$ the lowest singlet state $S_{1}-B_{2}$ crosses the first repulsive triplet state $T_{4}-B_{1}$ at a calculated energy of $4.8 \mathrm{eV}$-slightly higher than the $4.66 \mathrm{eV}$ corresponding to $266 \mathrm{~nm}$. Furthermore, the laser wavelength at $266 \mathrm{~nm}$ is not short enough to promote molecules to the triplet state

$\mathrm{T}_{4}-\mathrm{B}_{1}$ to undergo direct dissociation. Thus, the proposed mechanism does not seem plausible.

It should be worthwhile to speculate why our results differ from those by Han. ${ }^{[16]}$ It might be caused by different conditions of the molecular beams adopted. In our system, a rotational temperature of about $100 \mathrm{~K}$ is obtained by fitting ro-vibrational band profiles of the ${ }^{1} \mathrm{~B}_{2}\left({ }^{1} \Sigma_{u}^{+}\right)$state of $\mathrm{CS}_{2} \cdot{ }^{\left[{ }^{[6]}\right.}$ Despite the lack of the rotational temperature reported, Han and coworkers employed a valve with a $0.20 \mathrm{~mm}$ diameter orifice, in contrast to the $0.6 \mathrm{~mm}$ valve used herein. Thus, under their conditions, the molecular beam apparently yields a much cooler rotational temperature, probably of about $10 \mathrm{~K}$. Peebles ${ }^{[37]}$ reported Fourier-transform microwave spectra of ${ }^{79} \mathrm{Br}$ bromobenzene which gave rise to rotational constants of $A=5667.750, B=994.9018$, and $C=846.2567 \mathrm{MHz}$. The moments of inertia correspond to $1.46 \times 10^{-38}, 8.42 \times 10^{-38}$, and $9.85 \times 10^{-38} \mathrm{~g} \mathrm{~cm}^{-2}$, respectively. Given the rotational energy, $E_{\mathrm{r}}=h^{2} j(j+1) / 8 \pi^{2} I_{\mathrm{m}}$ (where $h$ is Planck's constant, $I_{\mathrm{m}}$ the moment of inertia and $j$ the rotational quantum number), $j$ can maximally be populated up to 4 and 13 at respective temperatures of 10 and $100 \mathrm{~K}$. Therefore, based on the molecular rotation period, $t_{\mathrm{r}}=h / 2 \pi j B_{\mathrm{m}}$ (where $B_{\mathrm{m}}$ is the rotational constant), ${ }^{[38]}$ $t_{r}$ is about 7,41 , and $47 \mathrm{ps}$ for the three rotational degrees of freedom at a temperature of $10 \mathrm{~K}$, in contrast to 2,12 , and $14 \mathrm{ps}$ at $100 \mathrm{~K}$. The average $t_{\mathrm{r}}$ becomes 32 and $9.3 \mathrm{ps}$ at 10 and $100 \mathrm{~K}$ respectively, if the rotational period in each degree of freedom is weighted equally. Referring to the dissociation lifetime of 26 ps by Davidsson and coworkers, ${ }^{[20]}$ the different rotational periods clearly explain why different $\beta$ values are obtained. The maximum $j$ in the photodissociation also affects the subsequent fraction of translational energy release.

\subsection{Photodissociation of lodobenzene}

Photodissociation of iodobenzene at $266 \mathrm{~nm}$ yields iodine fragments, in which $\mathrm{I}$ and $\mathrm{I}^{*}$ are subsequently ionized by $(2+1)$ REMPI at 265.9 and $266.5 \mathrm{~nm}$ respectively. Their images are then acquired with a velocity imaging detection system. By analogy with analysis of the bromine fragments, the raw and reconstructed ion images of the spin-orbit ground state I and the excited state $I^{*}$ are shown in Figure 4 . It can easily be seen that the ground state I gives rise to two ring components, whereas $I^{*}$ yields only one. For quantitative analysis, their speed distributions are extracted from the reconstructed 

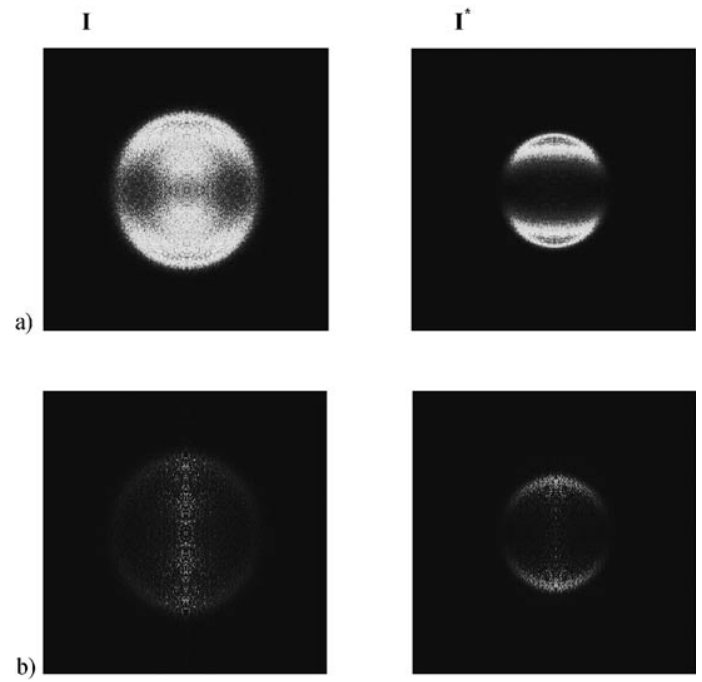

Figure 4. a) Raw ion image and b) inverse Abel-transformed ion image of the spin-orbit ground state $I$ and excited state $I^{*}$ for the photodissociation of iodobenzene at $266 \mathrm{~nm}$. The laser is linearly polarized in the vertical direction.

images, and converted to the center-of-mass translational energy distribution by using Equations (1) and (2), as shown in Figure 5. Given the $-\mathrm{C}_{6} \mathrm{H}_{5}$ bond dissociation energy of $66.7 \mathrm{kcal} \mathrm{mol}^{-1[39]}$ and the spin-obit spitting energy of $21.7 \mathrm{kcal}$ $\mathrm{mol}^{-1}$, the available energies $E_{\text {avl }}$ for the one-photon dissociation process are 40.8 and $18.8 \mathrm{kcal} \mathrm{mol}^{-1}$ in the I and I* fragmentations respectively, as marked in Figure 5. With the $\left\langle E_{\mathrm{T}}\right\rangle$ values estimated from the figure, the fractions of translational energy deposition are $26 \%$ and $70 \%$ for the slow and fast channels of the I fragment respectively, and $82 \%$ for the I* channel. These values are also listed in Table 1.

The branching ratio of I and I* can be estimated from the ratio of their measured ion signals by including a calibration factor, as expressed by

$\frac{N\left(I^{*}\right)}{N(\mathrm{I})}=k \frac{S\left(\mathrm{I}^{*}\right)}{S(\mathrm{I})}$

where $N\left(I^{*}\right)$ and $N(I)$ are the numbers of $I^{*}$ and I fragments; $S\left(I^{*}\right)$ and $S(I)$ are the ion intensities measured individually. The calibration factor $k$ is associated with the transition probability ratios in REMPI and related instrument factors. It is evaluated by performing a calibration experiment of $\mathrm{I}_{2}$ photolysis under the same conditions, and for the $266 \mathrm{~nm}$ photolysis of $\mathrm{I}_{2}, k$ is 0.455 . The branching ratio $N\left(I^{*}\right) / N(\mathrm{I})$ is 0.55 . The relative quantum yields of $\Phi\left(\mathrm{I}^{*}\right)$ and $\Phi(\mathrm{I})$ are calculated as 0.35 and 0.65 by using Equations (7) and (8):

$\Phi\left(I^{*}\right)=\frac{N\left(I^{*}\right)}{N(I)+N\left(I^{*}\right)}$

$\Phi(\mathrm{I})=1-\Phi\left(\mathrm{I}^{*}\right)$

Similar to the analysis adopted for the $\mathrm{Br}$ fragment, the iodine fragment's angular distribution is obtained from the reconstructed ion images. According to Equation (5), the aniso-
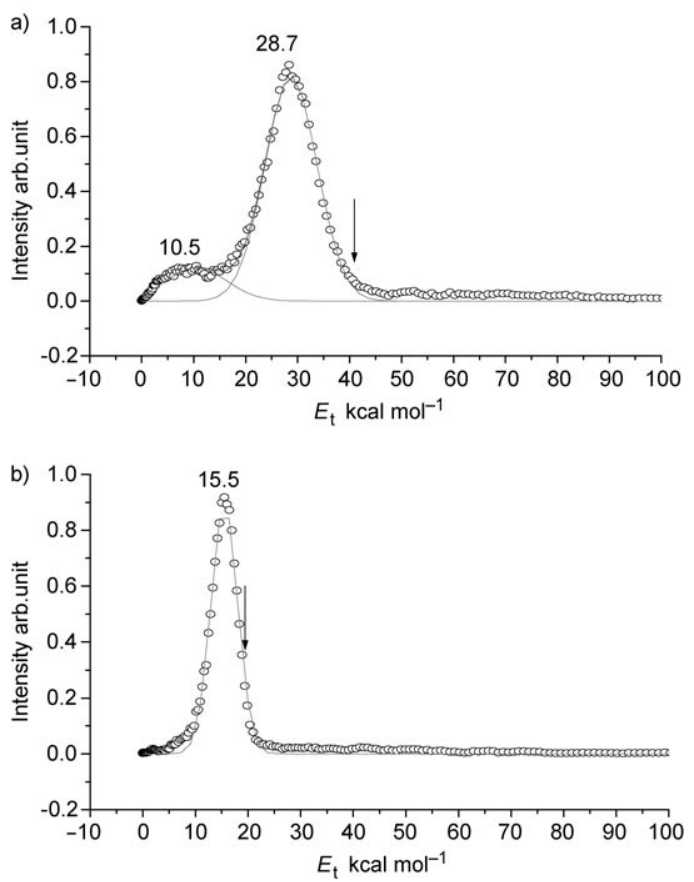

Figure 5. Center-of-mass translational energy distributions of a) the spinorbit ground state $I$ and $b$ ) the excited state $I^{*}$ fragments produced from photodissociation of iodobenzene at $266 \mathrm{~nm}$. The arrows indicate the maximum kinetic energy available for single-photon dissociation. Each data point (○) corresponds to the relative signal intensity in arbitrary units. In the I channel, the data points are deconvoluted into two Gaussian components denoted by (-). Their linear combination fits the experimental results. The data points in the $\mathrm{I}^{*}$ channel are fitted by a Gaussian function denoted by (—).

tropy parameter $\beta$ is 2.0 for the $\mathrm{I}^{*}$ fragmentation at $266 \mathrm{~nm}$, and $0.9 \pm 0.2$ and $0.7 \pm 0.2$ respectively, for the slow and fast dissociation channels leading to the I fragment. The positive value of $\beta$ suggests that the I and $\mathrm{I}^{*}$ fragments dissociate rapidly when the laser polarization direction is parallel to the $C-I$ bond axis.

By employing femtosecond pump-probe spectroscopy to analyze phenyl cation transients following the photodissociation of iodobenzene at $266 \mathrm{~nm}$, Kadi et al. obtained two dissociation lifetimes of 700 and $350 \mathrm{fs}^{[22]}$ The fast dissociation channel is ascribed to direct transition to a repulsive $\left(n-\sigma^{*}\right)$ state localized on the $\mathrm{C}-\mathrm{I}$ bond, whereas the slow one is related to an indirect dissociation by excitation to a bound singlet $\left(\pi-\pi^{*}\right)$ state in the phenyl ring which strongly couples to the repulsive $\left(n-\sigma^{*}\right)$ state and finally dissociates. About $57 \%$ of the molecules dissociate fast and the remaining ones undergo the slower predissociation. ${ }^{[22]}$ The dissociation times in both channels are essentially faster than a rotational period, in accord with our $\beta$ values for the iodine fragments. However, given the area ratio of the Gaussian components in Figure $5 \mathrm{a}$, we expect that $84 \%$ of the iodobenzene molecules may dissociate to the I fragment along the direct dissociation channel.

With the aid of one-dimensional potential energy curves along the $\mathrm{C}-\mathrm{I}$ bond dissociation, some insight into how the spin-orbit ground state and excited state iodine fragments are 
produced may be gained. As shown in Figure 3, a simplified energy diagram of potential energy curves referring to the calculations by Lunell and coworkers ${ }^{[25]}$ indicates the related dissociation pathways. The direct dissociation channel most likely involves the three repulsive states $S_{1}\left[\left(n-\sigma^{*}\right), B_{1}\right], T_{2}\left[\left(n-\sigma^{*}\right)\right.$, $\left.B_{1}\right]$, and $T_{5}\left[\left(n-\sigma^{*}\right), B_{2}\right]$ that have individual vertical excitation energies close to the laser excitation energy. The course of the direct photodissociation of iodobenzene is similar to that of the alkyl halides. For instance, the A-band spectra of $\mathrm{CH}_{3} \mathrm{I}$ containing three dissociative optical transitions may lead to fragmentation of $\mathrm{CH}_{3}$ and the spin-orbit ground $1\left({ }^{2} \mathrm{P}_{3 / 2}\right)$ or excited $I^{*}\left({ }^{2} \mathrm{P}_{1 / 2}\right)$ states. ${ }^{[40,41]}$ Among them, the ${ }^{3} \mathrm{Q}_{0}\left(2 \mathrm{~A}_{1}\right)$ state correlates to the $I^{*}$ fragment, while the ${ }^{3} \mathrm{Q}_{1}(2 \mathrm{E})$ and ${ }^{1} \mathrm{Q}_{1}(3 \mathrm{E})$ states correlate to I. When ${ }^{3} \mathrm{Q}_{0}\left(2 \mathrm{~A}_{1}\right)$ crosses to ${ }^{1} \mathrm{Q}_{1}(3 \mathrm{E})$, both I and ${ }^{*}$ may be obtained, depending on the state-coupling strength. Analogously, the singlet $S_{1}\left[\left(n-\sigma^{*}\right), B_{1}\right]$ state may correlate to the $I^{*}$ product in the photodissociation of iodobenzene, while the triplet $T_{5}\left[\left(n-\sigma^{*}\right), B_{2}\right]$ and $T_{2}\left[\left(n-\sigma^{*}\right), B_{1}\right]$ states correlate with the product $I$. Excitation of the singlet $S_{1}\left[\left(n-\sigma^{*}\right), B_{1}\right]$ state, which crosses the $T_{5}\left[\left(n-\sigma^{*}\right), B_{2}\right]$ state at the energy of 3.7-4.0 eV ${ }^{[25]}$ may lead to production of I and $I^{*}$, both of which are characteristic of the parallel transition. Inspection of the energy states in Figure 3 shows that vertical excitation to the $T_{5}\left[\left(n-\sigma^{*}\right), B_{2}\right]$ state would be difficult. Thus, $I^{*}$ is mostly obtained from the direct excitation of $S_{1}\left[\left(n-\sigma^{*}\right), B_{1}\right]$. This explains why only the parallel transition contributes to $\beta\left(I^{*}\right)$ obtained to be 2.0. If the $T_{2}\left[\left(n-\sigma^{*}\right), B_{1}\right]$ state is excited, only the I fragment is produced. Therefore, the positive value of $\beta(\mathrm{I})$ is due to the mixed characteristics of the nonadiabatic transition stemming from the $S_{1}$ state on the one hand, and the perpendicular transition from the $T_{2}$ state on the other. Note that either route of direct dissociation can cause the rapid rupturing of the $\mathrm{C}-\mathrm{I}$ bond such that most available energy may partition into the translational states, amounting to $70 \%$ and $82 \%$

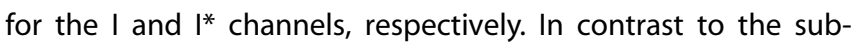
stantial branching of $\mathrm{I}^{*}$ found by us, El-Sayed and coworkers obtained only a small amount of $0.5 \%$ at $304 \mathrm{~nm} .{ }^{[14]}$ This might be because lowering the excitation energy results in a smaller probability to reach the $S_{1}$ state. Thus, excitation of the $\mathrm{T}_{2}$ state may dominate the direct dissociation channel.

For the predissociation channel, the iodobenzene molecules are excited to the bound singlet $S_{2}\left[\left(\pi-\pi^{*}\right), B_{2}\right]$ state, followed by strong coupling to the repulsive states prior to dissociation. At $266 \mathrm{~nm}, \mathrm{~T}_{2}\left[\left(\mathrm{n}-\sigma^{*}\right), \mathrm{B}_{1}\right], \mathrm{T}_{5}\left[\left(\mathrm{n}-\sigma^{*}\right), \mathrm{B}_{2}\right]$, and $\mathrm{S}_{1}\left[\left(\mathrm{n}-\sigma^{*}\right)\right.$, $B_{1}$ ] are the three probable states which cross the $S_{2}$ state at a point below the excitation energy. Thus, the predissociation mechanism can proceed rapidly. While considering dominance of the $1\left({ }^{2} \mathrm{P}_{3 / 2}\right)$ fragment in this channel, we expect that the bound singlet $S_{2}\left[\left(\pi-\pi^{*}\right), B_{2}\right]$ state strongly couples to the repulsive $T_{2}\left[\left(n-\sigma^{*}\right), B_{1}\right]$ and $T_{5}\left[\left(n-\sigma^{*}\right), B_{2}\right]$ states, which correlate with the ground state product. Since $S_{2}$ and $T_{5}$ have the same symmetry, the adiabatic transition $\left(S_{2} \rightarrow T_{5}\right)$ should let the $\mathrm{T}_{5}$ state have properties of both the parallel and the perpendicular transitions. The positive value of $\beta$ is attributed to the partial contribution of the parallel transition. As displayed in Figure 3 , the triplet-singlet coupling between $T_{5}\left[\left(n-\sigma^{*}\right), B_{2}\right]$ and $S_{1}\left[\left(n-\sigma^{*}\right), B_{1}\right]$ at $\sim 2.4 \AA$ prior to dissociation leads to the production of both I and I*. However, it is still unknown why the quantum yield of $\mathrm{I}^{*}$ in this channel is almost zero, except for a tiny contribution around $5-10 \mathrm{kcal} \mathrm{mol}^{-1}$ (Figure 5). Although the dissociative and predissociative mechanisms for the iodobenzene were first proposed a decade ago, ${ }^{[11,13,14,24]}$ the work herein with the aid of ab initio calculations may shed some light on further understanding of the photolysis of iodobenzene at $266 \mathrm{~nm}$.

\subsection{Comparison between Bromobenzene and lodobenzene}

Between bromobenzene and iodobenzene, the larger iodine atom has a marked influence on the stabilization of the repulsive excited states and the enhancement of spin-orbit coupling to facilitate the predissociation mechanism. According to the calculations by Lunell and coworkers, ${ }^{[25]}$ the vertical excitation energies for both singlet and triplet repulsive states $\left(n-\sigma^{*}\right)$ are lowered by $0.76-1.13 \mathrm{eV}$, enough for the curve crossing the bound singlet state to move to the lower energy levels. In this sense, there are two repulsive triplet states and one singlet state that cross to the bound singlet state of iodobenzene close to the excitation energy at $266 \mathrm{~nm}$, leading to more complicated dissociation routes. In contrast, the first repulsive triplet state of bromobenzene crosses the bound singlet state at about $0.14 \mathrm{eV}$ higher than the excitation energy, so the predissociative process is slowed and most of the available energy partitions into the internal states. ${ }^{[25]}$ The choice of halogen thus causes significant variation in dissociation mechanisms and lifetimes, the anisotropic nature of the halogen fragment, and the fractions of energy depositions.

\section{Conclusions}

As an alternative to the methods of femtosecond laser spectroscopy and photofragment translational spectroscopy, we utilized velocity imaging detection combined with $(2+1)$ REMPI to investigate photodissociation of bromobenzene and iodobenzene at $266 \mathrm{~nm}$. We proposed that bromobenzene follows a predissociative channel via singlet-triplet coupling. The dissociation mechanism is relatively slow, so that the $\mathrm{Br}$ fragment has an isotropic feature and most of its available energy partitions into the internal states. The ground state $\mathrm{Br}$ dominates the dissociation channel. In contrast, for the photodissociation of iodobenzene, the iodine substitution stabilizes the repulsive states along the $1-\mathrm{C}_{6} \mathrm{H}_{5}$ bond dissociation such that three repulsive curves can cross the bound singlet state at an energy level below the laser excitation energy. Thus, we proposed more complicated dissociation mechanisms for iodobenzene. The predissociative route is similar to that followed by bromobenzene, but the bound state-to-repulsive state couplings are much more efficient, resulting in rapid dissociation with a positive anisotropy parameter and a large fraction of translational energy deposition. As the major dissociation channel, direct dissociation can take place when the repulsive singlet or triplet states are excited. Both I and I* fragments are produced, relying on the spin-orbit coupling between these states. In this channel, the obtained anisotropy parameter is positive-similar 
to that of the predissociative mechanism-but a larger fraction of translational energy is partitioned from the available energy.

\section{Acknowledgements}

Z.R. and Y.T. acknowledge the financial support of IAMS and the Chinese Foundation for a visit to Professor Lin. This work is supported by the National Taiwan University, Ministry of Education, the National Science Council of Taiwan and the Republic of China under contract no. NSC 96-2113-M-002-027.

Keywords: halogens - photochemistry - resonance-enhanced multiphoton ionization - reactive intermediates $\cdot$ velocity imaging

[1] L. J. Butler, E. J. Hintsa, S. F. Shane, Y. T. Lee, J. Chem. Phys. 1987, 86, 2051-2074.

[2] D. L. Phillips, B. A. Lawrence, J. J. Valentini, J. Phys. Chem. 1991, 95, 9085-9091.

[3] B. R. Johnson, C. Kittrell, P. B. Kelly, J. L. Kinsey, J. Phys. Chem. 1996, 100 7743-7764.

[4] O. V. Rattigan, D. E. Shallcross, R. A. Cox, J. Chem. Soc. Faraday Trans. 1997, 93, 2839-2846.

[5] P. C. Samartzis, B. L. Bakker, D. H. Parker, T. N. Kitsopoulos, J. Phys. Chem. A 1999, 103, 6106-6113.

[6] G. Wu, B. Jiang, Q. Ran, J. Zhang, S. A. Harich, X. Yang, J. Chem. Phys. 2004, 120, 2193-2198.

[7] Y. Tang, L. Ji, R. Zhu, Z. Wei, B. Zhang, ChemPhysChem 2005, 6, $2137-$ 2144.

[8] Y. Tang, W. B. Lee, Z. Hu, B. Zhang, K. C. Lin, J. Chem. Phys. 2007, 126, 064302-064309.

[9] M. Dzvonik, S. Yang, R. Bersohn, J. Chem. Phys. 1974, 61, 4408-4421.

[10] A. Freedman, S. C. Yang, M. Kawasaki, R. Bersohn, J. Chem. Phys. 1980, $72,1028-1033$.

[11] P. Y. Cheng, D. Zhong, A. H. Zewail, Chem. Phys. Lett. 1995, 237, 399405.

[12] T. Ichimura, Y. Mori, H. Shinohara, N. Nishi, J. Chem. Phys. 1997, 107, 835-842.

[13] H. J. Hwang, M. A. El-Sayed, J. Chem. Phys. 1992, 96, 856-858.

[14] J. A. Griffiths, K. Junk, M. A. El-Sayed, J. Phys. Chem. 1996, 100, 79897996.

[15] S. Unny, Y. Du, L. Zhu, K. Truhins, R. J. Gordan, A. Sugita, M. Kawasaki, Y. Matsumi, R. Delmdahl, D. H. Parker, A. Berces, J. Phys. Chem. A 2001, $105,2270-2280$
[16] H. Zhang, R. S. Zhu, G. J. Wang, K. L. Han, G. Z. He, N. Q. Lou, J. Chem. Phys. 1999, 110, 2922-2927.

[17] X. B. Gu, G. J. Wang, J. H. Huang, K. L. Han, G. Z. He, N. Q. Lou, J. Phys. Chem. A 2001, 105, 354-362.

[18] M. Kadi, E. Ivarsson, J. Davidsson, Chem. Phys. Lett. 2004, 384, 35-39.

[19] Y. J. Liu, P. Persson, H. O. Karlsson, S. Lunell, M. Kadi, D. Karlsson, J. Davidsson, J. Chem. Phys. 2004, 120, 6502-6509.

[20] O. A. Borg, Y. J. Liu, P. Persson, S. Lunell, D. Karlsson, M. Kadi, J. Davidsson, J. Phys. Chem. A 2006, 110, 7045-7056.

[21] T. Ichimura, Y. Mori, H. Shinohara, N. Nishi, Chem. Phys. 1994, 189, $117-$ 125.

[22] M. Kadi, J. Davidsson, A. N. Tarnovsky, M. Rasmusson, E. Akesson, Chem Phys. Lett. 2001, 350, 93-98.

[23] W. H. Pence, S. L. Baughcum, S. R. Leone, J. Phys. Chem. 1981, 85, 3844 3851.

[24] J. E. Freitas, H. J. Hwang, M. A. El-Sayed, J. Phys. Chem. 1995, 99, 73957406.

[25] Y. J. Liu, P. Persson, S. Lunell, J. Phys. Chem. A 2004, 108, 2339-2345.

[26] C. L. Huang, J. C. Jiang, A. M. Mebel, Y. T. Lee, C. K. Ni, J. Am. Chem. Soc 2003, 125, 9814-9820.

[27] S. H. Lee, C. Y. Wu, S. K. Yang, Y. P. Lee, J. Chem. Phys. 2006, 125, 144301-7.

[28] R. S. Zhu, H. Zhang, G. J. Wang, X. B. Gu, K. L. Han, G. Z. He, N. Q. Lou, Chem. Phys. 1999, 248, 285-292.

[29] H. Zhang, R. S. Zhu, G. J. Wang, K. L. Han, G. Z. He, N. O. Lou, Chem. Phys. Lett. 1999, 300, 483-488.

[30] M. D. Poulsen, E. Skovsen, H. Stapelfeldt, J. Chem. Phys. 2002, 117, 2097-2102.

[31] E. Péronne, M. D. Poulsen, H. Stapelfeldt, C. Z. Bisgaard, E. Hamilton, T. Seideman, Phys. Rev. A 2004, 70, 063410-9.

[32] A. T. J. B. Eppink, D. H. Parker, Rev. Sci. Instrum. 1997, 68, 3477-3484.

[33] D. A. Dahl, J. E. Delmore, A. D. Appelhans, Rev. Sci. Instrum. 1990, 61, 607-609.

[34] R. N. Zare, Mol. Photochem. 1972, 4, 1-37.

[35] O. A. Borg, Chem. Phys. Lett. 2007, 436, 57-62.

[36] Z. Hu, W. B. Lee, X. P. Zhang, P. Y. Wei, K. C. Lin, ChemPhysChem 2008, 9, 422-430.

[37] S. A. Peebles, P. A. Peebles, J. Mol. Struct. 2003, 657, 107-116.

[38] R. D. Levine, R. B. Bernstein, Molecular Reaction Dynamics and Chemical Reactivity, Oxford University Press, New York, 1987, p. 284.

[39] S. S. Kumaran, M. C. Su, J. V. Michael, Chem. Phys. Lett. 1997, 269, 99106.

[40] A. T. J. B. Eppink, D. H. Parker, J. Chem. Phys. 1999, 110, 832-844.

[41] H. Xu, Y. Guo, S. Liu, X. Ma, D. Dai, G. Sha, J. Chem. Phys. 2002, 117, 5722-5729.

Received: December 14, 2007

Revised: March 14, 2008

Published online on April 25, 2008 